\title{
Latest advances in discontinuous deformation analysis method
}

\author{
JIAO YuYong ${ }^{1,2 *}$, ZHAO Qiang ${ }^{2}$, ZHENG Fei $^{2}$ \& WANG Long ${ }^{2}$ \\ ${ }^{1}$ Faculty of Engineering, China University of Geosciences, Wuhan 430074, China; \\ ${ }^{2}$ State Key Laboratory of Geomechanics and Geotechnical Engineering, Institute of Rock and Soil Mechanics, Chinese Academy of Sciences, \\ Wuhan 430071, China
}

Received August 29, 2016; accepted November 11, 2016; published online December 2, 2016

$\begin{array}{ll}\text { Citation: } & \text { Jiao Y Y, Zhao Q, Zheng F, et al. Latest advances in discontinuous deformation analysis method. Sci China Tech Sci, 2017, 60: 963-964, doi: } \\ \text { 10.1007/s11431-016-0634-y }\end{array}$

Discontinuous deformation analysis (DDA) method, proposed firstly by Shi [1] in 1988, is a novel numerical approach to simulate the discontinuous deformation behaviors of blocky rock structures. In DDA, the domain of interest is represented as an assemblage of discrete blocks and the joints are treated as interfaces between blocks. The governing equations of DDA are derived from Newton's Second Law of Motion and the Principle of Minimum Potential Energy. In the calculation, the large displacement solution of a block is obtained through the procedure of time-step integration, and accordingly, the collapse process of a block system can be reproduced dynamically. As no compatibility condition is needed to form the global equilibrium equations and each individual block moves independently, the calculation does not encounter mathematical problems even solving any large displacement.

As a theoretically rigorous numerical method, DDA has been one of the focuses in the discontinuous computing field since its birth. So far, the two-dimensional programs and related engineering applications are becoming mature. However, when the DDA method is extended from $2 \mathrm{D}$ to $3 \mathrm{D}$, and when it is used to solve large scale engineering problems, several major difficulties appear. These difficulties lie in three aspects, i.e., there is no accurate and robust contact theory to tackle 3D problems; the computational efficiency is low and the computational scale is relatively small; refining DDA

*Corresponding author (email: yyjiao@whrsm.ac.cn) simulation needs further improvements in basic theory and hybrid algorithms. Fortunately, aiming at the problems mentioned above, significant progresses have been made recently.

The most important advance in DDA study is the appearing of a novel approach for contact calculation. In 2015, Shi [2] proposed a general contact theory for $2 \mathrm{D}$ and $3 \mathrm{D}$ discontinuous computation, including a new definition for operating the point sets named as the entrance block. The boundary of an entrance block is a contact cover system. Each contact cover defines a contact point and all closed-contact points define the movements, rotations and deformations of all blocks as in real cases. Given a reference point, the concept of entrance block can simplify the contact computation greatly by determining the shortest distance between two blocks in a straightforward way, by defining the contact points through the first entrance, and by obtaining the shortest path of exit easily. As a robust method, the proposed new contact theory can help us in eliminating the biggest obstacle in 3D DDA programming.

There are also major improvements in basic DDA theory these years. To avoid the introduction of virtual springs that are commonly used in the classical DDA, Zheng et al. [3] proposed the dual form DDA in which the contact forces are utilized as the basic variables instead of the block displacements. Based on the projection-contraction algorithm, they designed the compatibility iteration for the quasi-variational inequality. The main advantage of this method is that the computational accuracy, robustness and efficiency can be guaranteed with removal of the virtual springs. In order to improve the ac- 
curacy of the disk-based DDA, Amir Reza Beyabanaki and Bagtzoglou [4] presented the new formulations of stiffness and force matrices for non-rigid disks using a new efficient contact model. In their model, disk-disk and disk-boundary contacts are transformed into the form of point-to-line contacts and normal spring, shear spring and frictional force sub-matrices are derived by vector analysis. The augmented Lagrangian method is used as the penalty function, and the reference line can be obtained directly by using only coordinates of disk centers and their radii. By introducing a viscous damping component to absorb discrete blocks' kinetic energy, Jiang et al. [5] established the global equations of the discrete block system that take damping effects into account, and defined the convergence criteria for DDA solutions, which provided more objective standards for the DDA application in geotechnical engineering.

Recent efforts in coupling DDA with other numerical methods are also noteworthy. Combining the FE-based rock failure process analysis (RFPA) method with the DDA method, Tang et al. [6] proposed a discontinuous deformation and displacement (DDD) analysis method for modeling the rock failure process. In the proposed model, RFPA is used to simulate crack initiation, propagation and coalescence processes of rock, while DDA is used to simulate the movement of blocks created by the multiple cracks. DDD method can automatically simulate crack propagation and block movement during the rock failure process without degrading the accuracy. Different from Tang's model, Choo et al. [7] introduced FE meshes into DDA computation and proposed a hydromechanical analysis algorithm to simulate fracturing problems initiated by hydraulic pressure. Through several simulation cases for verification, it is proved that the FEM-DDA hybrid model is reliable in modeling continuum-discontinuum problems. To improve the block deformability, Fu et al. [8] developed the dual reciprocity boundary element method (DRBEM) with stepwise updating to make it possible for the introduction of BE mesh into DDA. The advantage lies in its adoption of static fundamental solutions and reduction in the size of the governing equations by transforming the inertial term domain integrals to boundary integrals in the dynamic large displacement analysis.

Last but not least, the progress in DDA structural programming and verification cannot be ignored. As professional programmers, Cheng et al. [9] proposed a new software architecture for 3D DDA, and a structural source code with a user-friendly graphical interface was developed. To verify 3D DDA program, Chen et al. [10] performed a series of centrifuge tests to study the slope toppling mechanism, and compared the physical results with the simulated ones. Their findings proved the effectiveness of 3D DDA.

By studying the-state-of-the-art, three trends for DDA research can be predicted. Firstly, the present practical engineering is calling for high-performance numerical simulation, there is great need for improving the computational efficiency and size, thus, the parallel DDA algorithms for GPU and cloud computing are urgently requested. Secondly, because the real geotechnical projects are three-dimensional, we look forward to the commercial-level 3D DDA programs in not far future. Thirdly, besides the computational geomechanics, Shi's new contact theory is expected to deal with problems of other fields such as structure, mechanics and robotics.

1 Shi G H. Discontinuous deformation analysis-A new numerical model for the statics and dynamics of block systems. Dissertation of Doctoral Degree. Berkeley: University of California, 1988

2 Shi G H. Contact theory. Sci China Tech Sci, 2015, 58: 1450-1496

3 Zheng H, Zhang P, Du X. Dual form of discontinuous deformation analysis. Comput Method Appl M, 2016, 305: 196-216

4 Amir Reza Beyabanaki S, Bagtzoglou A C. Non-rigid disk-based DDA with a new contact model. Comput Geotech, 2013, 49: 25-35

5 Jiang Q, Chen Y, Zhou C, et al. Kinetic energy dissipation and convergence criterion of discontinuous deformations analysis (DDA) for geotechnical engineering. Rock Mech Rock Eng, 2013, 46: 1443-1460

6 Tang C A, Tang S B, Gong B, et al. Discontinuous deformation and displacement analysis: From continuous to discontinuous. Sci China Tech Sci, 2015, 58: 1567-1574

7 Choo L Q, Zhao Z, Chen $\mathrm{H}$, et al. Hydraulic fracturing modeling using the discontinuous deformation analysis (DDA) method. Comput Geotech, 2016, 76: 12-22

8 Fu G Y, Ma G W, Qu X L. Dual reciprocity boundary element based block model for discontinuous deformation analysis. Sci China Tech Sci, 2015, 58: 1575-1586

9 Cheng X L, Xiao J, Miao Q H, et al. Design and implementation of a software architecture for 3D-DDA. Sci China Tech Sci, 2015, 58: 1604-1608

10 Chen Z Y, Gong W J, Ma G W, et al. Comparisons between centrifuge and numerical modeling results for slope toppling failure. Sci China Tech Sci, 2015, 58: 1497-1508 